\title{
Association between serum $\beta 2$-microglobulin levels and frailty in an elderly Chinese population: results from RuLAS
}

\author{
This article was published in the following Dove Press journal: \\ Clinical Interventions in Aging \\ 16 October 2017 \\ Number of times this article has been viewed
}

\section{Zu-yun Liu',* \\ Yan-yu Shen ${ }^{2, *}$ \\ Li-juan $\mathrm{Ji}^{3}$ \\ Xiao-yan Jiang ${ }^{4}$ \\ Xiao-feng Wang ${ }^{5}$ \\ Yan $\mathrm{Shi}^{6}$}

'Department of Internal Medicine, Yale School of Medicine, New Haven, CT, USA; ${ }^{2}$ Department of Nephrology, People's Hospital of Xuyi, Xuyi, Jiangsu, China; ${ }^{3}$ Department of Sport Medicine and Pain Clinic, Center of Sports Rehabilitation, School of Sport Science, Shanghai University of Sport, ${ }^{4}$ Key Laboratory of Arrhythmias of the Ministry of Education of China, Tongji University School of Medicine, ${ }^{5}$ Unit of Epidemiology, Ministry of Education Key Laboratory of Contemporary Anthropology, School of Life Sciences, Fudan University, Shanghai, China, ${ }^{6}$ Department of Emergency, Huai'an Second People's Hospital, The Affiliated Huai'an Hospital of Xuzhou Medical University, Huai'an, China

*These authors contributed equally to this work
Objective: To examine the association between serum $\beta 2$-microglobulin (B2M) levels and frailty in an elderly Chinese population.

Design: A population-based cohort study.

Setting and participants: We used data on 1,663 elderly participants (aged 70-84 years) from the aging arm of the Rugao Longevity and Ageing study, a population-based observational two-arm cohort study conducted in Rugao, China.

Measurements: The serum B2M was measured with chemiluminescence immunoassay by a technician in the biochemistry laboratory of the Rugao People's Hospital. Information on the frailty index and phenotype was collected.

Results: The mean B2M levels and frailty index were $1.8 \mathrm{mg} / \mathrm{L}$ and 0.16 , respectively; 188 (11.3\%) participants were classified as frail (frailty phenotype). For a standard deviation increase in B2M, the adjusted odds ratio for frailty phenotype was 1.20 (95\% CI: $1.05,1.39 ; P=0.009)$ and the standardized coefficient for frailty index was 0.07 (95\% CI: $0.02,0.11 ; P=0.004)$. Relative to the lowest quartile, the highest B2M quartile had a greater risk of prevalent frailty with adjusted odds ratios of 1.68 (95\% CI: 1.04, 2.71; $P=0.034)$ for frailty phenotype and 1.51 (95\% CI: 1.01 , $2.27 ; P=0.044)$ for frailty index ( $\geq 0.25$ ). In addition, estimated glomerular filtration rate (based on $\mathrm{B} 2 \mathrm{M}$ ) or chronic kidney disease (estimated glomerular filtration rate $<60 \mathrm{~mL} / \mathrm{min} / 1.73 \mathrm{~m}^{2}$ ) was significantly associated with frailty phenotype and index.

Conclusion: B2M was significantly associated with both frailty phenotype and index in elderly Chinese population. The findings underscore the promising kidney relevant biomarkers for identifying vulnerable elderly Chinese population.

Keywords: frailty phenotype, frailty index, elderly, kidney, biomarker

\section{Introduction}

Frailty is an important geriatric syndrome that is associated with adverse health outcomes such as hospitalization and mortality. ${ }^{1}$ Identifying the related factors of frailty (eg, blood biomarkers) would provide a better understanding of underlying mechanisms and clues that could be crucial for public health interventions. To date, serum 32 -microglobulin (B2M), a novel kidney filtration marker, ${ }^{2}$ shows satisfactory predictive utility of all-cause mortality, specific disease-related mortality, and other health outcomes (eg, end-stage renal disease) across a wide range of populations. ${ }^{3}$ A recent review summarized the important role of $\mathrm{B} 2 \mathrm{M}$ in immune surveillance and modulation, multiple diseases, and malignancy. ${ }^{4}$ Given the close relationship between chronic kidney disease and frailty, ${ }^{5,6}$ one typical multisystem syndrome, it is intuitive and important to evaluate the association between B2M and frailty.

\footnotetext{
Correspondence: Yan Shi

Department of Emergency, Huai'an Second People's Hospital, The Affiliated Huai'an Hospital of Xuzhou Medical University, Huai'an, China

Tel +86 I59 96197560

Email jmfsy@I63.com
} 
To the best of our knowledge, only two relevant studies have been conducted that suggested association between B2M levels and frailty in French and Japanese populations, ${ }^{7,8}$ which underscores the clinical prognostic value of B2M. However, little is known about this relationship in elderly Chinese population, one fastest growing segment of the population in this largest developing country. More importantly, the disparity in these persons' socioeconomic status, lifestyles, and psychosocial stress over the past few decades has resulted in variations in their aging processes. Much attention has been drawn to the pattern of chronic conditions and relevant factors in this unique population, for example, testing whether the observed association in other populations would hold. In addition, the only two previous studies have some limitations. For example, one of them was a pilot study and another only included women. ${ }^{7,8}$ Therefore, based on data from a population-based observational cohort study, this study aimed to investigate the association between serum B2M levels and frailty in an elderly Chinese population.

\section{Methods}

\section{Study population}

We used data from the aging arm of the Rugao Longevity and Ageing Study, a 2-arm cohort study conducted in Rugao, Jiangsu Province, China. A detailed description is provided elsewhere. ${ }^{9}$ Briefly, we randomly recruited 1960 elderly adults (aged 70-84 years) from 31 villages of Jiang' an township, Rugao, between November and December 2014 according to a 5-year age and sex strata. We finally recruited 1,788 participants (91.2\%). ${ }^{9}$ This was a community-based study with no exclusion criteria. ${ }^{9}$ No substantial difference in the 5-year age and sex ratios was found between the responders and nonresponders. Out of the 1,788 participants, 125 participants were excluded from the current study due to missing data (eg, frailty), leaving a final study sample of 1,663 participants. ${ }^{10}$

During the field investigation, a detailed structured questionnaire and physical examination were administered by trained physicians from the Rugao People's Hospital. We collected information on sociodemographics, chronic diseases (eg, cerebrovascular disease), lifestyles, health deficits (eg, urinary incontinence), and sleep quality. Routine clinical examinations (eg, electrocardiography and blood pressure) and a walking test (timed "up and go" test, TUG) were also performed. Over $98 \%$ of all participants provided fasting blood samples. Each participant provided written informed consent. This study was approved by the Human Ethics Committee of Fudan University School of Life Sciences.

\section{Serum B2M}

Participants provided fasting blood samples in the morning during the field investigation. The serum B2M was measured with chemiluminescence immunoassay by a technician in the biochemistry laboratory of the Rugao People's Hospital. The estimated glomerular filtration rate (eGFR) was calculated using the same equation as in previous studies. ${ }^{7}$

\section{Frailty}

To date, two main approaches have been used to operationalize frailty based on different theories, including the phenotype model and the cumulative deficit model..$^{1,1-13} \mathrm{We}$ included these two approaches in this study.

\section{Frailty phenotype}

According to Fried et $\mathrm{al}^{11}$ five criteria used to define the frailty phenotype include unintentional weight loss, exhaustion, low activity, slowness, and weakness. In this study, we used similar measurements of the five criteria and the detailed description has been provided in previous reports. ${ }^{14,15}$ In brief, unintentional weight loss, exhaustion, and low activity were based on self-reported items, including "weight has decreased by $4.5 \mathrm{~kg}$ or $5 \%$ during the last 12 months", "feeling tired all of the time (at least 3 or 4 days a week)", and "needing help to walk". Slowness was defined as being below the 20th sex-specific percentile in gait speed (assessed by a TUG test). In the TUG test, the study participants were asked to stand up from an armchair, walk $3 \mathrm{~m}$, return, and sit down again. The timing of this test began when the participants' back came off the back of the armchair and stopped when their buttocks touched the seat of the chair again. ${ }^{16}$ Weakness was based on the self-report defined as "having difficulty in lifting or carrying something as heavy as $10 \mathrm{~kg}$ ", which was similar to that used in previous studies. ${ }^{17}$ Participants who met with three or more of the five criteria were categorized as having frailty.

\section{Frailty index}

According to the standard procedure suggested by Rockwood and Mitnitski ${ }^{12}$ and Searle et al, ${ }^{13} 45$ health deficits that include symptoms, comorbidities, activities of daily living (basic and instrumental), cognitive and psychological function were used to construct a frailty index in this study, and the detailed description was provided in our previous publication. ${ }^{18}$ In brief, we dichotomized or polychotomized each deficit and mapped it to the interval $0-1$ to represent the severity of this deficit. Then frailty index was calculated by summing all deficits and dividing by the total number of 
deficits $(n=45)$, with a range from 0 to 1 . According to Song et al, ${ }^{19}$ participants were categorized as having frailty if they had a frailty index $\geq 0.25$.

\section{Statistical analyses}

Descriptive data were presented as means (SD) or numbers (percentage). The chi-square test and the Mann-Whitney test (the Kruskal-Wallis test when appropriate) were used for comparisons of characteristics according to B2M quartiles. To examine the associations of B2M with frailty phenotype and index, logistic regression models and/or linear regression models were performed. In this study, two models were used. Model 1 was an unadjusted model, whereas model 2 was adjusted for covariates such as age, sex, marital status, education level, smoking and drinking habits, body mass index, number of comorbidities (only for frailty phenotype). We repeated the above analyses for men and women separately. In addition, considering the possible impact of kidney diseases (eg, kidney stones, and renal cyst) or cancer on B2M levels, we repeated the above analyses in those without these conditions. A significant difference was defined as $P<0.05$ (2-tailed). We performed all statistical analyses using SAS software, version 9.4 (SAS Institute Inc., Cary, NC, USA).

\section{Results}

Table 1 presents characteristics of the study participants according to B2M levels. The mean B2M levels and frailty index were $1.8 \mathrm{mg} / \mathrm{L}$ and 0.16 , respectively; 188 (11.3\%) of the study participants were classified as frail (frailty phenotype). From B2M quartile 1-4, the proportions of participants with frailty slightly increased. For example, the proportions of participants who had frailty defined by frailty phenotype were $7.6 \%$ in quartile $1,10.8 \%$ in quartile $2,11.3 \%$ in quartile 3 , and $15.7 \%$ in quartile 4 .

Table 2 shows the association between serum B2M levels, kidney function, and frailty. For a standard deviation increase in $\mathrm{B} 2 \mathrm{M}$, the adjusted odds ratio (OR) for frailty phenotype was 1.20 (95\% CI: $1.05,1.39 ; P=0.009)$ and the standardized coefficient for frailty index was 0.07 (95\% CI: 0.02, 0.11; $P=0.004)$. Relative to the lowest quartile, the highest B2M quartile had a greater risk of prevalent frailty with adjusted ORs of 1.68 (95\% CI: $1.04,2.71 ; P=0.034)$ for frailty phenotype and $1.51(95 \% \mathrm{CI}: 1.01,2.27 ; P=0.044)$ for frailty index $(\geq 0.25)$. In addition, eGFR (per $10 \mathrm{~mL} / \mathrm{min} / 1.73 \mathrm{~m}^{2}$ ) or chronic kidney disease (eGFR $<60 \mathrm{~mL} / \mathrm{min} / 1.73 \mathrm{~m}^{2}$ ) was significantly associated with frailty phenotype and index. No substantial difference in the associations was found between men and women. In addition, we did not find substantial changes in the associations between B2M and frailty in those without kidney diseases or cancer. For example, in those without kidney diseases $(n=1,644)$, for a standard deviation increase in B2M, the OR for frailty phenotype was $1.20(95 \%$ CI: $1.05,1.38 ; P=0.010)$ and the standardized coefficient for frailty index was 0.07 (95\% CI: 0.02, $0.12 ; P=0.003)$.

\section{Discussion}

In this study, we found that B2M was significantly associated with both frailty phenotype and index in elderly Chinese population. To our knowledge, no prior study has examined the relationship in this unique population.

Table I Characteristics of the study participants according to B2M levels in an elderly Chinese population

\begin{tabular}{|c|c|c|c|c|c|c|}
\hline \multirow[t]{2}{*}{ Characteristic } & \multirow{2}{*}{$\begin{array}{l}\text { Overall } \\
(n=1,663)\end{array}$} & \multicolumn{4}{|c|}{ B2M level (mg/L) } & \multirow[t]{2}{*}{$P$-value ${ }^{c}$} \\
\hline & & $\begin{array}{l}\text { Quartile I } \\
(<I .5, n=422)\end{array}$ & $\begin{array}{l}\text { Quartile } 2 \\
(I .5-1.7, n=426)\end{array}$ & $\begin{array}{l}\text { Quartile } 3 \\
(1.8-2.1, n=408)\end{array}$ & $\begin{array}{l}\text { Quartile } 4 \\
(\geq 2.2, n=407)\end{array}$ & \\
\hline Age, years, mean (SD) & $75.3(3.9)$ & $74.1(3.4)$ & $74.7(3.7)$ & $75.6(3.8)$ & $76.9(4.0)$ & $<0.001$ \\
\hline Female, n (\%) & $885(53.2)$ & $239(56.6)$ & $227(53.3)$ & $2 I \mid(5 I .7)$ & $208(5 I .1)$ & 0.380 \\
\hline Currently married, n (\%) & $1,094(65.8)$ & $284(67.3)$ & $286(67.1)$ & $268(65.7)$ & $256(62.9)$ & 0.516 \\
\hline Illiterate, n (\%) & $895(53.8)$ & $234(55.5)$ & $214(50.2)$ & $213(52.2)$ & $234(57.5)$ & 0.152 \\
\hline Smoker (ever), n (\%) ${ }^{\mathrm{a}}$ & $424(25.5)$ & $100(23.7)$ & I I0 (25.8) & $105(25.7)$ & $109(26.8)$ & 0.775 \\
\hline Drinker (ever), n (\%) & $488(29.3)$ & $134(3 \mid .8)$ & I2I (28.4) & I2I (29.7) & II $2(27.5)$ & 0.565 \\
\hline Body mass index, $\mathrm{kg} / \mathrm{m}^{2}$, mean (SD) & $24.1(3.5)$ & $24.1(3.4)$ & $24.0(3.1)$ & $23.9(3.7)$ & $24.3(3.7)$ & 0.647 \\
\hline No of comorbidities, mean (SD) ${ }^{\mathrm{b}}$ & $1.5(1.2)$ & $1.5(1.1)$ & $\mathrm{I} .4(\mathrm{I} . \mathrm{I})$ & $\mathrm{I} .5(\mathrm{I} . \mathrm{I})$ & $\mathrm{I} .7(\mathrm{I} .2)$ & 0.011 \\
\hline $\mathrm{B} 2 \mathrm{M}, \mathrm{mg} / \mathrm{L}$, mean $(\mathrm{SD})$ & $\mathrm{I} .8(0.7)$ & $1.3(0.2)$ & $1.6(0.1)$ & $1.9(0.1)$ & $2.7(0.8)$ & $<0.001$ \\
\hline Frailty defined by frailty phenotype, n (\%) & $188(\mid 1.3)$ & $32(7.6)$ & $46(10.8)$ & $46(11.3)$ & $64(15.7)$ & 0.003 \\
\hline Frailty index, mean (SD) & $0.16(0.09)$ & $0.16(0.09)$ & $0.16(0.09)$ & $0.16(0.08)$ & $0.18(0.09)$ & 0.005 \\
\hline Frailty index $\geq 0.25, \mathrm{n}(\%)$ & $254(15.3)$ & $53(12.6)$ & $65(15.3)$ & $49(12.0)$ & $87(21.4)$ & $<0.001$ \\
\hline
\end{tabular}

Notes: aParticipants were categorized as smokers (ever) if they responded "Yes" to the question "Have you ever smoked continuously for longer than 6 months?"; Drinker (ever) was defined in the same way. 'Comorbidities included hypertension, diabetes, coronary artery disease, cerebrovascular disease, chronic lung disease, osteoporosis, arthritis, eye disease, fractures, thyroid disease, arrhythmia, Parkinson's disease, and cancer. 'For continuous and categorical variables, the chi-square test and the MannWhitney test (the Kruskal-Wallis test when appropriate) were used, respectively.

Abbreviation: B2M, 32 -microglobulin. 
Table 2 Association between serum B2M levels, kidney function and frailty in an elderly Chinese population

\begin{tabular}{|c|c|c|c|c|c|c|c|c|}
\hline & \multicolumn{4}{|c|}{ Frailty phenotype } & \multicolumn{4}{|l|}{ Frailty index } \\
\hline & \multicolumn{2}{|l|}{ Model I' } & \multicolumn{2}{|l|}{ Model $2^{b}$} & \multicolumn{2}{|l|}{ Model I ${ }^{\mathrm{b}}$} & \multicolumn{2}{|l|}{ Model $2^{b}$} \\
\hline & OR $(95 \% \mathrm{CI})$ & $P$-value & OR $(95 \% \mathrm{Cl})$ & $P$-value & $\begin{array}{l}\text { Coefficient or } \\
\text { OR }(95 \% \mathrm{Cl})\end{array}$ & $P$-value & $\begin{array}{l}\text { Coefficient or } \\
\text { OR }(95 \% \mathrm{Cl})\end{array}$ & $P$-value \\
\hline \multicolumn{9}{|l|}{ B2M level } \\
\hline Per SD increase ${ }^{a}$ & $1.33(1.16,1.53)$ & $<0.001$ & $1.20(1.05,1.39)$ & 0.009 & $0.10(0.05,0.15)^{c}$ & $<0.001$ & $0.07(0.02,0.11)^{c}$ & 0.004 \\
\hline $\begin{array}{l}\text { Quartile (mg/L) } \\
\text { (reference quartile I, <I.5) }\end{array}$ & Ref & - & Ref & - & Ref & - & Ref & - \\
\hline $2(1.5-1.7)$ & I.48 $(0.92,2.37)$ & 0.107 & I.43 $(0.88,2.34)$ & 0.150 & $1.25(0.85,1.85)^{d}$ & 0.257 & $1.20(0.80,1.80)^{d}$ & 0.388 \\
\hline $3(1.8-2.1)$ & $1.55(0.97,2.49)$ & 0.070 & $1.38(0.85,2.26)$ & 0.197 & $0.95(0.63,1.44)^{d}$ & 0.810 & $0.84(0.55,1.30)^{d}$ & 0.435 \\
\hline $4(\geq 2.2)$ & $2.27(1.45,3.56)$ & $<0.001$ & I.68 (I.04, 2.7I) & 0.034 & I.89 $(1.30,2.75)^{d}$ & $<0.001$ & $1.5 \mathrm{I}(\mathrm{I} .0 \mathrm{I}, 2.27)^{\mathrm{d}}$ & 0.044 \\
\hline \multicolumn{9}{|l|}{ eGFR } \\
\hline $\begin{array}{l}\text { Per } 10 \mathrm{~mL} / \mathrm{min} / 1.73 \mathrm{~m}^{2} \\
\text { decrease, continuous }\end{array}$ & $1.20(\mathrm{I} .1 \mathrm{I}, \mathrm{I} .30)$ & $<0.001$ & $1.14(1.05,1.24)$ & 0.002 & $1.13(1.07,1.22)^{d}$ & $<0.001$ & $1.10(1.02,1.18)^{d}$ & 0.013 \\
\hline$<60 \mathrm{~mL} / \mathrm{min} / 1.73 \mathrm{~m}^{2}$ & $2.27(I .5 I, 3.4 I)$ & $<0.001$ & $1.84(1.19,2.83)$ & 0.006 & $1.98(1.36,2.89)^{d}$ & $<0.001$ & $1.63(1.09,2.45)^{d}$ & 0.018 \\
\hline
\end{tabular}

Notes: aB2M was transformed to Z-scores with a mean of 0 and SD of I. bModel I is an unadjusted model; model 2 adjusted for age, sex, marital status, education level, smoking and drinking habits, body mass index, and number of comorbidities (only for frailty phenotype). "Frailty index was transformed to Z-scores with a mean of 0 and

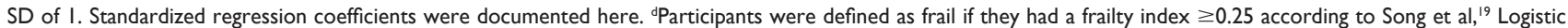
regression models were used and $\mathrm{ORs}(95 \% \mathrm{Cl})$ were documented.

Abbreviations: B2M, $\beta 2$-microglobulin; eGFR, estimated glomerular filtration rate; OR, odds ratio.

The findings provide preliminary but important data for relevant research, and raised much concern about kidney dysfunction in frail elderly.

Cystatin C, a serum measure of renal function, is a stronger predictor of the risk of death and cardiovascular events in the elderly than is creatinine. ${ }^{20}$ However, it is reported that B2M shares cystatin C's advantage over serum creatinine, predicting adverse outcomes across a wide range of population. ${ }^{3}$ The identified association of B2M with frailty in this study is consistent with previous reports, ${ }^{7,8}$ further underscoring the importance of $\mathrm{B} 2 \mathrm{M}$ and kidney dysfunction in frail elderly populations. Many studies have demonstrated that chronic kidney disease accelerates the metabolic abnormalities of aging such as chronic inflammation, oxidative stress, and protein energy wasting, resulting in a high risk of frailty. ${ }^{5,6,21-23}$ There might be other possible explanations of the identified association, which require future explorations.

This study contributes to the current literature in several aspects. First, two common frailty measurements that are based on different theories of frailty and therefore identify different risk sets were employed. ${ }^{17,24,25}$ We compared them in this cohort and found a satisfied agreement with a slight difference. ${ }^{10,17}$ Their similar associations with $\mathrm{B} 2 \mathrm{M}$ in this study may imply common mechanisms underlying frailty. Second, this study included large sample size with men and women, extending previous findings to general Chinese populations. ${ }^{7,8}$ Third, the findings are important when considering the study population had unique characteristics, such as a higher proportion of illiterate participants relative to previous reports. ${ }^{7}$ In fact, the unique features also include highly prevalent hypertension and diabetes. These public issues have evoked a large number of studies, especially focusing on the rapidly growing vulnerable elderly populations.

Nevertheless, the cross-section design in this study impedes causal inference. Although Kim et al reported a slight association between B2M and incident frailty with longitudinal data, ${ }^{7}$ the power is limited to draw a scientific conclusion. Second, we modified components for frailty phenotype despite no substantial influence in literature. But our subsequent studies demonstrated that the modification did not substantially influence the predictive utility of adverse outcomes. ${ }^{10,14,15}$ Third, the wide $95 \%$ CI of ORs and marginally significant differences in the proportion of frail persons across the four quartiles suggest that the study sample size is still limited, particularly for research on frailty phenotype.

In summary, we demonstrated that B2M was associated with frailty in an elderly Chinese population, which further underscores the promising kidney relevant biomarkers for identifying vulnerable elderly Chinese population. Future studies with longitudinal assessments of B2M and frailty are particularly needed to validate our findings and promote a better understanding of the relationship between kidney function and frailty.

\section{Acknowledgments}

We thank Dr George Agogo for his help with the writing. We are thankful for the comments from the two anonymous 
reviewers. Dr Liu is the recipient of a James Hudson BrownAlexander B Coxe Fellowship from Yale University School of Medicine, and he also received support from U01AG022376. This work was supported by Grants 81571372 and 81670465 from the National Natural Science Foundation and the Yale Claude D. Pepper Older Americans Independence Center (P30AG021342).

\section{Disclosure}

The authors report no conflicts of interest in this work.

\section{References}

1. Clegg A, Young J, Iliffe S, Rikkert MO, Rockwood K. Frailty in elderly people. Lancet. 2013;381(9868):752-762.

2. Filler G, Priem F, Lepage N, et al. Beta-trace protein, cystatin C, beta(2)microglobulin, and creatinine compared for detecting impaired glomerular filtration rates in children. Clin Chem. 2002;48(5):729-736.

3. Foster MC, Inker LA, Levey AS, et al. Novel filtration markers as predictors of all-cause and cardiovascular mortality in US adults. Am J Kidney Dis. 2013;62(1):42-51.

4. Li L, Dong M, Wang XG. The implication and significance of beta 2 microglobulin: a conservative multifunctional regulator. Chin Med $J$ (Engl). 2016;129(4):448-455.

5. Chowdhury R, Peel NM, Krosch M, Hubbard RE. Frailty and chronic kidney disease: a systematic review. Arch Gerontol Geriatr. 2017;68: 135-142.

6. Shlipak MG, Stehman-Breen C, Fried LF, et al. The presence of frailty in elderly persons with chronic renal insufficiency. Am J Kidney Dis. 2004;43(5):861-867.

7. Kim M, Suzuki T, Kojima N, et al. Association between serum beta2-microglobulin levels and prevalent and incident physical frailty in community-dwelling older women. J Am Geriatr Soc. 2017;65(4): e83-e88.

8. Annweiler C, Bataille R, Ferriere N, Douillet D, Fantino B, Beauchet O. Plasma beta-2 microglobulin as a marker of frailty in older adults: a pilot study. J Gerontol A Biol Sci Med Sci. 2011;66(10):1077-1079.

9. Liu Z, Wang Y, Zhang Y, et al. Cohort Profile: The Rugao Longevity and Ageing Study (RuLAS). Int J Epidemiol. 2016;45(4):1064-1073.

10. Liu Z, Wang Q, Zhi T, et al. Frailty index and its relation to falls and overnight hospitalizations in elderly Chinese people: a population-based study. J Nutr Health Aging. 2016;20(5):561-568.
11. Fried LP, Tangen CM, Walston J, et al. Frailty in older adults: evidence for a phenotype. J Gerontol A Biol Sci Med Sci. 2001;56(3):M146-M156.

12. Rockwood K, Mitnitski A. Frailty in relation to the accumulation of deficits. J Gerontol A Biol Sci Med Sci. 2007;62(7):722-727.

13. Searle SD, Mitnitski A, Gahbauer EA, Gill TM, Rockwood K. A standard procedure for creating a frailty index. BMC Geriatr. 2008;8:24.

14. Zhu Y, Liu Z, Wang Y, et al. Agreement between the frailty index and phenotype and their associations with falls and overnight hospitalizations. Arch Gerontol Geriatr. 2016;66:161-165.

15. Liu Z, Burgess S, Wang Z, et al. Associations of triglyceride levels with longevity and frailty: A Mendelian randomization analysis. $S_{c i}$ Rep. 2017;7:41579.

16. Nordin E, Lindelof N, Rosendahl E, Jensen J, Lundin-Olsson L. Prognostic validity of the timed up-and-go test, a modified get-upand-go test, staff's global judgement and fall history in evaluating fall risk in residential care facilities. Age Ageing. 2008;37(4):442-448.

17. Blodgett J, Theou O, Kirkland S, Andreou P, Rockwood K. Frailty in NHANES: Comparing the frailty index and phenotype. Arch Gerontol Geriatr. 2015;60(3):464-470.

18. Liu Z, Wang Q, Zhi T, et al. Frailty index and its relation to falls and overnight hospitalizations in elderly Chinese people: a population-based study. J Nutr Health Aging. 2016;20(5):561-568.

19. Song X, Mitnitski A, Rockwood K. Prevalence and 10-year outcomes of frailty in older adults in relation to deficit accumulation. $J$ Am Geriatr Soc. 2010;58(4):681-687.

20. Shlipak MG, Sarnak MJ, Katz R, et al. Cystatin C and the risk of death and cardiovascular events among elderly persons. N Engl J Med. 2005;352(20):2049-2060.

21. Walker SR, Wagner M, Tangri N. Chronic kidney disease, frailty, and unsuccessful aging: a review. J Ren Nutr. 2014;24(6):364-370.

22. Portilla Franco ME, Tornero Molina F, Gil Gregorio P. Frailty in elderly people with chronic kidney disease. Nefrologia. 2016;36(6):609-615.

23. Dalrymple LS, Katz R, Rifkin DE, et al. Kidney function and prevalent and incident frailty. Clin J Am Soc Nephrol. 2013;8(12):2091-2099.

24. Orkaby AR, Hshieh TT, Gaziano JM, Djousse L, Driver JA. Comparison of two frailty indices in the physicians' health study. Arch Gerontol Geriatr. 2017;71:21-27.

25. Kulminski AM, Ukraintseva SV, Kulminskaya IV, Arbeev KG, Land K, Yashin AI. Cumulative deficits better characterize susceptibility to death in elderly people than phenotypic frailty: lessons from the Cardiovascular Health Study. J Am Geriatr Soc. 2008;56(5):898-903.
Clinical Interventions in Aging

\section{Publish your work in this journal}

Clinical Interventions in Aging is an international, peer-reviewed journal focusing on evidence-based reports on the value or lack thereof of treatments intended to prevent or delay the onset of maladaptive correlates of aging in human beings. This journal is indexed on PubMed Central, MedLine,

\section{Dovepress}

CAS, Scopus and the Elsevier Bibliographic databases. The manuscript management system is completely online and includes a very quick and fair peer-review system, which is all easy to use. Visit http://www.dovepress. com/testimonials.php to read real quotes from published authors. 